\title{
In-Hospital Blood Pressure Variability: A Novel Prognostic Marker of Renal Function Decline and Cardiovascular Events in Patients with Coronary Artery Disease
}

\author{
Kan Saito Yuichi Saito Hideki Kitahara Takashi Nakayama \\ Yoshihide Fujimoto Yoshio Kobayashi \\ Department of Cardiovascular Medicine, Chiba University Graduate School of Medicine, \\ Chiba, Japan
}

\section{Keywords}

In-hospital blood pressure variability · Cardiovascular events · Renal function decline

\begin{abstract}
Introduction: Several measures of blood pressure (BP) variability have been associated with kidney disease and cardiovascular events. Although BP is routinely measured during hospitalization in daily practice, the prognostic impact of in-hospital BP and its variability are uncertain. Methods: A total of 226 participants who underwent elective percutaneous coronary intervention $(\mathrm{PCl})$ for stable coronary artery disease $(C A D)$ were included. BP was measured by trained nurses during the 4-day hospitalization for PCI. BP variability was assessed by standard deviation (SD) and coefficient variation of systolic BP. Estimated glomerular filtration rate (eGFR) was calculated at baseline and follow-up ( $\geq 6$ months). The cardiovascular end point was defined as a composite of cardiovascular death, acute coronary syndrome, stroke, heart failure hospitalization, and any coronary revascularization. Results: In-hospital BP was measured $9.5 \pm 0.8$ times. During a median follow-up period of 1.7 years, mean eGFR change was $-1.7 \mathrm{~mL} / \mathrm{min} / 1.73 \mathrm{~m}^{2}$ per year, and 35 (15.5\%) participants met the cardiovascular end point. Mean systolic BP and SD were negatively correlated with eGFR change. In the receiver operating characteristic curve analysis, SD of systolic BP predicted the cardiovascular end point (AUC 0.63 , best cutoff value $14.2 \mathrm{~mm} \mathrm{Hg}, p=0.003$ ). Kaplan-Meier analysis demonstrated a significantly higher incidence of the cardiovascular end point in patients with SD of systolic $\mathrm{BP} \geq 14.2 \mathrm{~mm} \mathrm{Hg}$ compared to their counterpart $(p=0.003$ ). A multivariable analysis showed SD of systolic BP as an independent predictor for the cardiovascular end point. When assessed with coefficient variation, BP variability was similarly related to eGFR change and clinical outcomes. Conclusion: Greater in-hospital BP variability was associated with renal function decline and cardiovascular events in patients with stable CAD.




\section{Introduction}

Blood pressure (BP) is a dynamic physiological parameter, and hypertension is a leading cause for cardiovascular disease and mortality $[1,2]$. To date, several types of BP variability, such as diurnal [3], day-by-day [4], and visit-to-visit BP variability [5], have been reported to associate with future cardiovascular events beyond absolute BP levels [6]. In addition, visitto-visit BP variability has been associated with renal function decline [7-11]. These measures of variability are evaluated via ambulatory monitoring, at home, or in outpatient clinics; however, BP is also routinely measured during hospitalization. Recently, several studies, mostly including patients with stroke, have indicated that in-hospital BP variability is related to worse clinical outcomes [12-15]. However, the prognostic impact of in-hospital BP variability on renal function and clinical outcomes in other high cardiovascular risk patients is unclear. The aim of the present study was to assess the relationship between in-hospital BP variability and renal function decline or future cardiovascular events in patients with stable coronary artery disease (CAD).

\section{Material and Methods}

\section{Study Population}

This was a retrospective, single-center, observational study reported according to the Strengthening the Reporting of Observational Studies in Epidemiology (STROBE) statement. Between April 2013 and December 2014, a total of 662 percutaneous coronary intervention (PCI) procedures were performed in 481 patients at Chiba University Hospital. Patients were considered eligible for the present study when PCI procedures were electively performed for stable CAD. All PCI procedures were guided by intravascular ultrasound $[16,17]$. In our institution, patients usually underwent elective PCI during 4-day hospitalization. The major exclusion criteria were acute coronary syndrome, duplicated patients, no follow-up of renal function, atrial fibrillation, PCI performed during hospitalization for reasons other than stable CAD, and hemodialysis (Fig. 1). All patient characteristics at baseline were obtained from

Fig. 1. Study flow chart. BP, blood pressure; PCI, percutaneous coro-

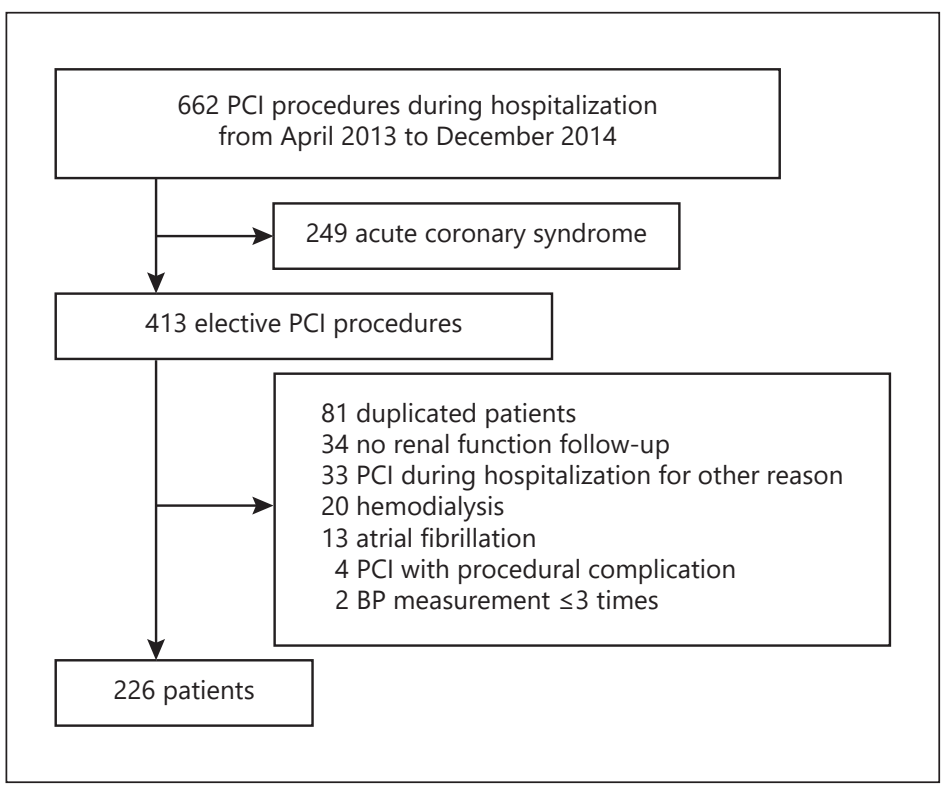
nary intervention. 


\section{Research}

Saito et al.: Impact of In-Hospital BP Variability

Table 1. Baseline characteristics

\begin{tabular}{lc}
\hline Variable & All $(n=226)$ \\
\hline Age, years & $67.7 \pm 10.2$ \\
Men, $n(\%)$ & $185(82)$ \\
BMI, kg/m ${ }^{2}$ & $23.8 \pm 3.5$ \\
Hypertension, $n(\%)$ & $175(77)$ \\
Diabetes mellitus, $n(\%)$ & $91(40)$ \\
Dyslipidemia, $n$ (\%) & $174(77)$ \\
Current smoker, $n(\%)$ & $47(21)$ \\
eGFR, mL/min/1.73 m ${ }^{2}$ & $68.7 \pm 18.7$ \\
Prior myocardial infarction, $n(\%)$ & $86(38)$ \\
Prior coronary artery bypass graft, $n(\%)$ & $11(5)$ \\
Medical treatment, $n(\%)$ & \\
Antiplatelet & $224(99)$ \\
Anticoagulant & $10(4)$ \\
Statin & $196(87)$ \\
ACE-I or ARB & $147(65)$ \\
$\beta$-blocker & $131(58)$ \\
Calcium channel blocker & $114(50)$ \\
Diuretics & $47(21)$ \\
PCI procedures, $n(\%)$ & \\
Drug-eluting stent use & $220(97)$ \\
IVUS guidance & $226(100)$ \\
Chronic total occlusion & $20(9)$ \\
In-stent restenosis & $19(8)$ \\
Number of treated vessels & $1.9 \pm 0.7$ \\
$\quad$ LMT or LAD, $n(\%)$ & $105(46)$ \\
$\quad$ LCX, $n(\%)$ & $57(25)$ \\
$\quad$ RCA, $n(\%)$ & $70(31)$ \\
Total stent length, mm & $44.0 \pm 28.9$ \\
\hline
\end{tabular}

Data are presented as $n(\%)$ and mean \pm SD, unless otherwise stated. ACE-I, angiotensin-converting enzyme inhibitor; ARB, angiotensin receptor blocker; eGFR, estimate glomerular filtration rate; IVUS, intravascular ultrasound; LAD, left anterior descending artery; LCX, left circumflex artery; LMT, left main trunk; PCI, percutaneous coronary intervention; RCA, right coronary artery; SD, standard deviation.

medical records in which basic patient information (e.g., age, sex, height, and weight), risk factors (e.g., hypertension, diabetes, dyslipidemia, and smoking), past medical history, and medication were systematically collected by clinicians.

\section{$B P$ and Heart Rate Measurement}

Resting BP and heart rate (HR) were measured by trained nurses with patients in the supine position using an automated cuff sphygmomanometer (ES-H55; Terumo, Tokyo, Japan). BP and HR were usually measured 3 times per day for 4 days during hospitalization at 6:00, 14:00, and 17:00. On day 4, however, BP and HR were usually measured once only at 6:00. Patients with $\geq 4$ measurements of BP and HR were included in this study. In-hospital $\mathrm{BP}$ and HR variabilities were evaluated by standard deviation (SD) and coefficient of variation (CV) of systolic BP and HR [18]. CV was defined as the within-subject SD divided by BP or HR levels. Patient adherence to medications was strictly monitored during hospitalization by trained nurses. 
Table 2. Predictors of eGFR change

\begin{tabular}{|c|c|c|c|c|}
\hline \multirow[t]{2}{*}{ Variable } & \multicolumn{2}{|c|}{ Univariable } & \multicolumn{2}{|c|}{ Multivariable } \\
\hline & $R$ & $p$ value & $\beta$ & $p$ value \\
\hline Age (years) & -0.04 & 0.58 & & \\
\hline Men & -0.18 & 0.90 & & \\
\hline BMI $\left(\mathrm{kg} / \mathrm{m}^{2}\right)$ & 0.04 & 0.50 & & \\
\hline Hypertension & -0.09 & 0.18 & & \\
\hline Dyslipidemia & 0.04 & 0.59 & & \\
\hline Diabetes mellitus & -0.16 & 0.02 & -0.13 & 0.04 \\
\hline Current smoker & -0.07 & 0.29 & & \\
\hline Amount of administered contrast (mL) & -0.08 & 0.24 & & \\
\hline ACE-I or ARB & -0.01 & 0.87 & & \\
\hline Calcium channel blocker & -0.04 & 0.52 & & \\
\hline Diuretics & -0.16 & 0.01 & -0.19 & 0.004 \\
\hline Baseline eGFR (mL/min $\left./ 1.73 \mathrm{~m}^{2}\right)$ & -0.03 & 0.27 & -0.16 & 0.02 \\
\hline Mean systolic BP (mm Hg) & -0.22 & $<0.001$ & -0.17 & 0.01 \\
\hline SD of systolic BP & -0.21 & 0.002 & -0.16 & 0.02 \\
\hline
\end{tabular}

Baseline eGFR was included into multivariable model irrespective of a $p$ value on univariable analysis. ACE-I, angiotensin-converting enzyme inhibitor; ARB, angiotensin receptor blocker; BP, blood pressure; eGFR, estimated glomerular filtration rate; SD, standard deviation.

\section{Outcomes}

Follow-up data were ascertained from medical records at Chiba University Hospital. Serum $\mathrm{Cr}$ was measured at baseline and latest follow-up ( $\geq 6$ months) in routine clinical practice as an outpatient or inpatient. Estimated glomerular filtration rate (eGFR) at each time point was calculated using the Japanese coefficient-modified Chronic Kidney Disease Epidemiology Collaboration equation [19]. Renal function decline was expressed as eGFR change $\left(\mathrm{mL} / \mathrm{min} / 1.73 \mathrm{~m}^{2}\right.$ per year), which was calculated as the difference between eGFR at follow-up and baseline, divided by follow-up years. The primary end points were (1) the rate of renal function decline and (2) the cardiovascular end point defined as a composite of cardiovascular death, myocardial infarction, stroke, heart failure hospitalization, and any coronary revascularization.

\section{Statistical Analysis}

Statistical analysis was performed with EZR statistical software package version 3.4.1 (The R Foundation for Statistical Computing, Vienna, Austria) and SAS statistical software package version 9.4 (SAS Institute, Cary, NC, USA). No missing data were encountered in the present study (Tables 1-3). Data are expressed as mean \pm SD, median (interquartile range), or frequency (\%), as appropriate. Univariable analysis was performed using linear regression analysis of rank-transformed outcomes. The associated variables on univariable analysis $(p$ $<0.10$ ) were included in multivariable analysis. Mean systolic BP was included in the multivariable model irrespective of a $p$ value on univariable analysis to adjust SD of systolic BP. Baseline eGFR was included into multivariable analysis regardless of a $p$ value on univariable analysis in the eGFR change analysis. Multiple linear regression analysis of rank-transformed outcomes was used for multivariable analysis. Since CV of systolic BP was highly collinear with SD of systolic BP due to the calculation method, CV of systolic BP was not included into the multivariable model with SD of systolic BP. The receiver operating characteristic curve analyses were conducted based on the occurrence of cardiovascular end point(s). Thresholds 
Table 3. Cox proportional hazards analysis for cardiovascular end point

\begin{tabular}{|c|c|c|c|c|}
\hline \multirow[t]{2}{*}{ Variable } & \multicolumn{2}{|l|}{ Univariable } & \multicolumn{2}{|l|}{ Multivariable } \\
\hline & $\operatorname{HR}(95 \% \mathrm{CI})$ & $p$ value & HR (95\% CI) & $p$ value \\
\hline Age (years) & $1.00(0.97-1.03)$ & $>0.99$ & & \\
\hline Men & $0.61(0.29-1.30)$ & 0.20 & & \\
\hline $\mathrm{BMI}\left(\mathrm{kg} / \mathrm{m}^{2}\right)$ & $1.00(0.91-1.11)$ & 0.97 & & \\
\hline Hypertension & $2.13(0.75-6.04)$ & 0.16 & & \\
\hline Dyslipidemia & $0.85(0.41-1.77)$ & 0.66 & & \\
\hline Diabetes mellitus & $0.97(0.50-1.90)$ & 0.93 & & \\
\hline Current smoker & $0.51(0.18-1.43)$ & 0.20 & & \\
\hline Baseline eGFR (mL/min $\left./ 1.73 \mathrm{~m}^{2}\right)$ & $0.99(0.97-1.00)$ & 0.13 & & \\
\hline Calcium channel blocker & $1.08(0.56-2.10)$ & 0.82 & & \\
\hline Diuretics & $1.28(0.60-2.72)$ & 0.53 & & \\
\hline ACE-I or ARB & $1.80(0.82-3.95)$ & 0.15 & & \\
\hline Mean systolic BP (mm Hg) & $1.00(0.97-1.03)$ & 0.96 & $0.99(0.96-1.02)$ & 0.33 \\
\hline SD of systolic BP & $1.10(1.03-1.17)$ & 0.003 & $1.11(1.03-1.19)$ & 0.005 \\
\hline In-stent restenosis & $2.67(1.11-6.44)$ & 0.03 & $1.97(0.80-4.84)$ & 0.14 \\
\hline Chronic total occlusion & $2.18(0.84-5.64)$ & 0.11 & & \\
\hline Drug-eluting stent use & $0.40(0.12-1.32)$ & 0.14 & & \\
\hline Total stent length (mm) & $1.00(0.98-1.01)$ & 0.43 & & \\
\hline
\end{tabular}

Mean systolic BP was included into multivariable model irrespective of a $p$ value on univariable analysis to adjust SD of systolic BP. ACE-I, angiotensin-converting enzyme inhibitor; ARB, angiotensin receptor blocker; BP, blood pressure; CI, confidence intervals; eGFR, estimated glomerular filtration rate; HR, hazard ratio; $\mathrm{SD}$, standard deviation.

for SD of systolic BP were established by finding the values which corresponded to the maximum average sensitivity and specificity. Kaplan-Meier analysis with the log-rank test was used to compare event-free survival rates. A Cox proportional hazards model was used to estimate unadjusted and adjusted hazard ratios with corresponding 95\% confidence intervals. Since this was an exploratory study and no reports have investigated in-hospital BP variability in patients with CAD, sample size calculation was not performed. A value of $p<$ 0.05 was considered statistically significant.

\section{Results}

A total of 226 patients were included in this study. Median follow-up was 1.7 (0.9-3.3) years and all were followed-up for at least 6 months. Baseline characteristics are shown in Table 1. BP and HR were measured $9.5 \pm 0.9$ times during hospitalization. Mean systolic BP and HR were $122.4 \pm 14.0 \mathrm{~mm} \mathrm{Hg}$ and $67.7 \pm 9.2 \mathrm{bpm}$. SD of systolic BP and HR were $12.3 \pm$ $4.3 \mathrm{~mm} \mathrm{Hg}$ and $6.3 \pm 2.8 \mathrm{bpm}$. CV of systolic BP and HR were $10.1 \pm 3.2$ and $9.3 \pm 3.9$, respectively.

\section{Renal Outcome}

Mean eGFR change was $-1.7 \pm 8.9 \mathrm{~mL} / \mathrm{min} / 1.73 \mathrm{~m}^{2}$ per year. Table 2 showed significant negative correlations of diabetes mellitus, use of diuretics, mean systolic BP, and SD of systolic BP with eGFR change on the univariable analysis. On the other hand, mean HR and HR variability did not have a significant correlation with eGFR decline. Multivariable analysis demonstrated mean systolic BP and SD of systolic BP to be independent predictors of eGFR change, in addition to diabetes mellitus, use of diuretics, and baseline eGFR as shown in Table 2. CV of 


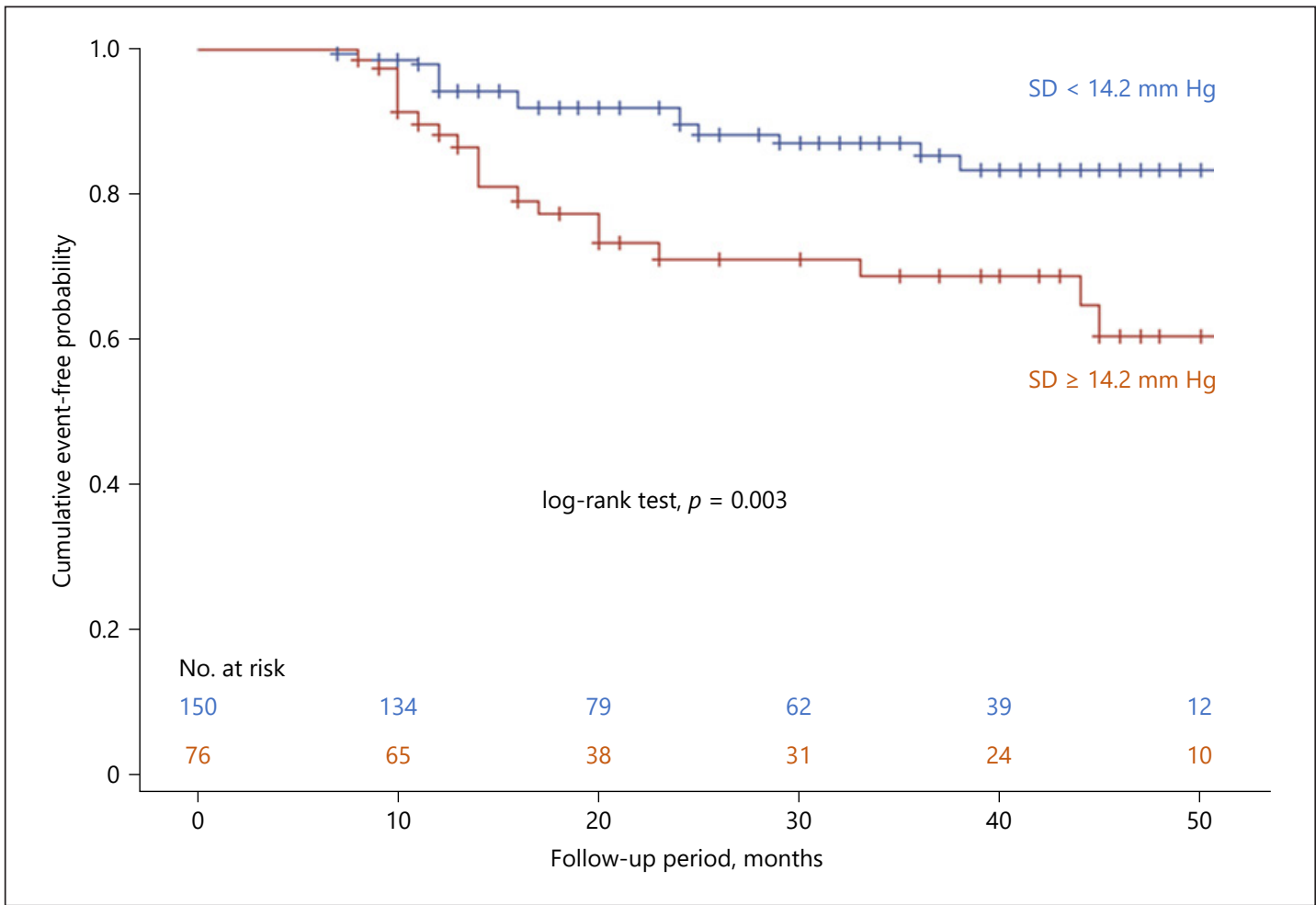

Fig. 2. Kaplan-Meier analysis for the probability of the cardiovascular end point consist of cardiovascular death, myocardial infarction, stroke, heart failure hospitalization, and any coronary revascularization in patient with SD of systolic BP $\geq 14.2 \mathrm{~mm} \mathrm{Hg}$ and the counterpart. SD, standard deviation; BP, blood pressure.

systolic BP was associated with eGFR change similarly to SD of systolic BP (online suppl. Table 2; for all online suppl. material, see www.karger.com/doi/10.1159/000509291). The ability in predicting eGFR change of each multivariable model was shown in online suppl. Table 3.

\section{Cardiovascular End Point}

Following PCI, 35 patients (15.5\%) reached the cardiovascular end point, including cardiovascular death $(n=4)$, myocardial infarction $(n=5)$, stroke $(n=2)$, heart failure hospitalization $(n=5)$, and any coronary revascularization $(n=28)$. In the receiver operating characteristic curve analyses, SD and CV of systolic BP predicted the cardiovascular end point (AUC 0.63, best cutoff value $14.2 \mathrm{~mm} \mathrm{Hg}, p=0.003$ and AUC 0.63 , best cutoff value 13.0, $p=$ 0.001 ), while mean systolic BP did not (AUC $0.52, p=0.86$ ). Patient and lesion characteristics in patients with SD of systolic BP less than and greater than or equal to $14.2 \mathrm{~mm} \mathrm{Hg}$ are shown in online suppl. Table 1. Kaplan-Meier analysis demonstrated a significantly higher incidence of the cardiovascular end points in patients with SD of systolic BP $\geq 14.2 \mathrm{~mm}$ Hg compared to those with lower BP variability (Fig. 2). A Cox proportional hazards regression analysis showed SD of systolic BP to be an independent predictor for the cardiovascular end point as shown in Table 3. CV of systolic BP was also associated with cardiovascular events similarly to SD of systolic BP (online suppl. Table 4). 


\section{Kidney \\ Blood Pressure \\ Research}

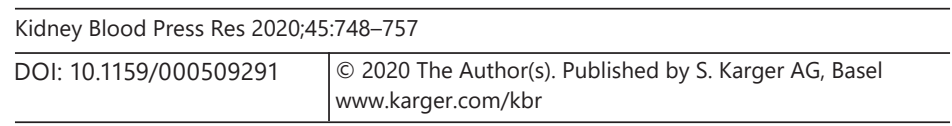

Saito et al.: Impact of In-Hospital BP Variability

\section{Discussion}

The present study demonstrated the potential prognostic utility of in-hospital BP variability on renal function and clinical outcomes in patients with stable CAD who undergoing PCI. Patients with greater in-hospital BP variability assessed by SD of systolic BP had higher risks of decline in eGFR and subsequent cardiovascular disease, independent of mean BP.

To date, several types of BP variability have been proposed and studied in the short term (e.g., diurnal) [3], medium term (e.g., day-by-day) [4], and long term (e.g., visit-to-visit) [5]. These variabilities are differently evaluated through ambulatory monitoring system, at home by the patients themselves, and in outpatient clinics by health care providers. Perhaps because of the different methods and periods of evaluation, these different measures of BP variability have shown a limited relationship with the other [20], and the prognostic value of these BP variabilities is not equivalent [21]. Recently, some studies, mostly including patients with acute stroke, have shown that BP variability during hospitalization (i.e., in-hospital BP) is associated with worse clinical outcomes [12-15]. However, little is known about the prognostic impact of in-hospital BP variability in patients with cardiovascular disease other than stroke. Although in-hospital BP variability may be similar to medium-term measures of dayto-day BP variability, the evaluation method is different. The day-to-day BP variability is assessed by BP at home measured once or twice per day for a few weeks $[4,17,22]$, whereas in-hospital BP variability is evaluated during hospitalization and measured by care providers $\geq 3$ times per day [12-15]. Since in-hospital BP is a simple index and readily available in daily clinical practice, risk stratification by in-hospital BP variability may be pragmatic and of clinical importance.

In the general population, GFR usually decreases by -1.0 to $-0.7 \mathrm{~mL} / \mathrm{min} / 1.73 \mathrm{~m}^{2}$ per year $[10,23]$, whereas annual eGFR change in the present study was $-1.7 \mathrm{~mL} / \mathrm{min} / 1.73 \mathrm{~m}^{2}$ per year. Several factors have been identified as predictors for progression of kidney disease, such as causes of CKD (e.g., diabetic nephropathy), age, sex, elevated BP, and a history of cardiovascular disease [23]. The population in the present study was at relatively high risk with established CAD. Thus, the absolute value of renal function decline in this study may be higher than those in the general population. Visit-to-visit BP variability is known to be associated with longitudinal deterioration of renal function [8-11]. A retrospective cohort study with a very long follow-up (i.e., 15 years) demonstrated a significant correlation of visit-tovisit BP variability assessed by SD of systolic BP in the clinics with eGFR change $(r=-0.16)$ [10], which was consistent with our results. Additionally, increased visit-to-visit BP variability is also associated with key measures of CKD progression, including doubling of serum Cr level, ESRD, and death [11]. The present study is the first, to our knowledge, to show a significant relation between in-hospital BP variability and renal function decline, independent of mean BP and known predictors of decline in renal function (diabetes and baseline renal function). It is conceivable that adherence to therapy is one of the causes of visit-to-visit BP variability [24]. However, medication adherence was strictly monitored during hospitalization in this study, thus, excluding this possibility. Other potential causes of in-hospital BP variability are differences in sympathetic drive, arterial compliance, genetic factors, and humoral regulation $[10,21,24]$. Clinicians could use increased in-hospital BP variability to identify a population at high-risk for deterioration in renal function, which itself is a powerful predictor of cardiovascular events [25].

In-hospital BP variability was associated with future renal function decline and clinical outcomes even though mean systolic BP (i.e., $122.4 \pm 14.0 \mathrm{~mm} \mathrm{Hg}$ ) was well controlled in the present study. BP variability has previously been associated with later cardiovascular events [6]. In line with our findings, several observational studies have recently indicated that the higher visit-to-visit BP variability in patients with CAD is a strong predictor for increased risk of cardio- 


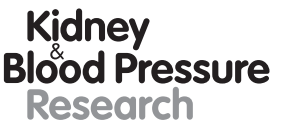

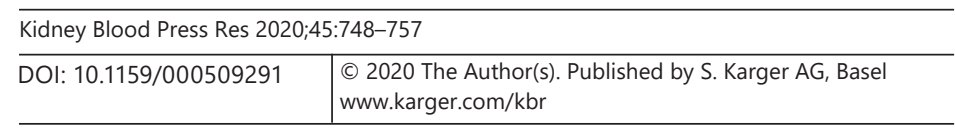

Saito et al.: Impact of In-Hospital BP Variability

vascular events, independent of mean BP [26,27]. There have been no validated cutoff values for $\mathrm{BP}$ variability that identify those at a significantly increased risk of cardiovascular events [10]. Nonetheless, CAD patients with increased in-hospital BP variability may benefit from more aggressive secondary preventive therapies (e.g., higher potency statin therapy) [28]. In addition, since it has been reported that drug-class effects on BP variability are different among antihypertensive drugs [29], the in-hospital BP variability might be a novel therapeutic target. Given the wide availability of in-hospital BP data in electronic health records, in-hospital BP variability represents a potentially promising area for clinical investigation.

There are some limitations in the present study. Follow-up data were obtained from medical records. Patients included in this study were all Japanese and $82 \%$ were male. Thus, caution is warranted in extrapolating the present study results in different populations. Some potential factors associated with renal function decline (e.g., urine albumin-to-Cr ratio and non-steroidal anti-inflammatory drug) and clinical outcomes (eGFR, drug-eluting stent use, and smoking) could not be included in the multivariable analyses because of the limited number of patients and missing variables. We used SD and CV to assess in-hospital BP variability in the present study, although other metrics such as average real variability may have different impact on renal function decline and cardiovascular end point. BP measurement could have been affected by the PCI procedure during hospitalization. Since 50 out of 226 patients underwent BP measurement, only $\leq 3$ times before PCI, BP-related parameters could not be compared between before and after PCI. BP and HR were measured during hospitalization, which may differ from those measured in the outpatient clinic or at home. Although in-hospital BP variability was significantly associated with renal and clinical outcomes in the present study, the predictivity was modest. There were no reports to investigate the relation between in-hospital BP variability and other measures of BP variability. Thus, further studies are warranted to clarify these relationships. In conclusion, greater in-hospital BP variability was associated with renal function decline and future cardiovascular events in patients undergoing PCI for stable CAD.

\section{Acknowledgements}

We gratefully acknowledge the work of past and present members of our laboratory.

\section{Statement of Ethics}

The research was conducted in accordance with the World Medical Association Declaration of Helsinki and complies with the guidelines for human studies. Written informed consent was obtained from all patients, and the Ethics Committee of Chiba University approved this study.

\section{Conflict of Interest Statement}

There are no conflicts of interest.

\section{Funding Sources}

No funding was received for this study. 


\section{Kidney \\ Blood Pressure \\ Research}

\begin{tabular}{l|l}
\hline Kidney Blood Press Res 2020:45:748-757 \\
\hline DOI: 10.1159/000509291 & $\begin{array}{l}\text { @ 2020 The Author(s). Published by S. Karger AG, Basel } \\
\text { www.karger.com/kbr }\end{array}$ \\
\hline
\end{tabular}

Saito et al.: Impact of In-Hospital BP Variability

\section{Author Contributions}

Conception and design: K. Saito, Y. Saito, and H. Kitahara. Drafting of the manuscript or critical revision for important intellectual content: K. Saito, Y. Saito, H. Kitahara, T. Nakayama, Y. Fujimoto, and Y. Kobayashi. Final approval of the submitted manuscript: K. Saito, Y. Saito, H. Kitahara, T. Nakayama, Y. Fujimoto, and Y. Kobayashi.

\section{References}

1 James PA, Oparil S, Carter BL, Cushman WC, Dennison-Himmelfarb C, Handler J, et al. 2014 evidence-based guideline for the management of high blood pressure in adults: report from the panel members appointed to the Eighth Joint National Committee (JNC 8). JAMA. 2014 Feb;311(5):507-20.

2 Lim SS, Vos T, Flaxman AD, Danaei G, Shibuya K, Adair-Rohani H, et al. A comparative risk assessment of burden of disease and injury attributable to 67 risk factors and risk factor clusters in 21 regions, 1990-2010: a systematic analysis for the Global Burden of Disease Study 2010. Lancet. 2012 Dec;380(9859):2224-60.

3 Kario K. Prognosis in relation to blood pressure variability: pro side of the argument. Hypertension. 2015 Jun; 65(6):1163-9.

4 Kikuya M, Ohkubo T, Metoki H, Asayama K, Hara A, Obara T, et al. Day-by-day variability of blood pressure and heart rate at home as a novel predictor of prognosis: the Ohasama study. Hypertension. 2008 Dec;52(6): 1045-50.

5 Rothwell PM, Howard SC, Dolan E, O’Brien E, Dobson JE, Dahlöf B, et al. Prognostic significance of visit-to-visit variability, maximum systolic blood pressure, and episodic hypertension. Lancet. 2010 Mar;375(9718):895905.

6 Stevens SL, Wood S, Koshiaris C, Law K, Glasziou P, Stevens RJ, et al. Blood pressure variability and cardiovascular disease: systematic review and meta-analysis. BMJ. 2016 Aug;354:i4098.

7 Gosmanova EO, Mikkelsen MK, Molnar MZ, Lu JL, Yessayan LT, Kalantar-Zadeh K, et al. Association of systolic blood pressure variability with mortality, coronary heart disease, stroke, and renal disease. J Am Coll Cardiol. 2016 Sep;68(13):1375-86.

8 Yokota K, Fukuda M, Matsui Y, Hoshide S, Shimada K, Kario K. Impact of visit-to-visit variability of blood pressure on deterioration of renal function in patients with non-diabetic chronic kidney disease. Hypertens Res. 2013 Feb;36(2):151-7.

9 Okada H, Fukui M, Tanaka M, Matsumoto S, Mineoka Y, Nakanishi N, et al. Visit-to-visit blood pressure variability is a novel risk factor for the development and progression of diabetic nephropathy in patients with type 2 diabetes. Diabetes Care. 2013 Jul;36(7):1908-12.

10 Chia YC, Lim HM, Ching SM. Long-term visit-to-visit blood pressure variability and renal function decline in patients with hypertension over 15 years. J Am Heart Assoc. 2016 Nov;5(11):e003825.

11 McMullan CJ, Lambers Heerspink HJ, Parving HH, Dwyer JP, Forman JP, de Zeeuw D. Visit-to-visit variability in blood pressure and kidney and cardiovascular outcomes in patients with type 2 diabetes and nephropathy: a post hoc analysis from the RENAAL study and the Irbesartan Diabetic Nephropathy Trial. Am J Kidney Dis. 2014 Nov;64(5):714-22.

12 Fukuda K, Kai H, Kamouchi M, Hata J, Ago T, Nakane H, et al. Day-by-day blood pressure variability and functional outcome after acute ischemic stroke: Fukuoka stroke registry. Stroke. 2015 Jul;46(7):1832-9.

13 Shi Z, Li ES, Zhong JS, Yuan JL, Li LR, Zheng CW. Predictive significance of day-to-day blood pressure variability in acute Ischemic stroke for 12-month functional outcomes. Am J Hypertens. 2017 May;30(5):524-31.

14 Kang J, Hong JH, Jang MU, Choi NC, Lee JS, Kim BJ, et al. Change in blood pressure variability in patients with acute ischemic stroke and its effect on early neurologic outcome. PLoS One. 2017 Dec;12(12):e0189216.

15 Duan Z, Tao L, Tang T, Fu C, Liu J, Chen B, et al. Effect of blood pressure variability on early neurological deterioration in single small subcortical infarction with parental arterial disease. eNeurologicalSci. 2017 Jun;8: 22-7.

16 Saito Y, Kobayashi Y, Fujii K, Sonoda S, Tsujita K, Hibi K, et al. Clinical expert consensus document on standards for measurements and assessment of intravascular ultrasound from the Japanese Association of Cardiovascular Intervention and Therapeutics. Cardiovasc Interv Ther. 2020 Jan;35(1):1-12.

17 Sonoda S, Hibi K, Okura H, Fujii K, Honda Y, Kobayashi Y. Current clinical use of intravascular ultrasound imaging to guide percutaneous coronary interventions. Cardiovasc Interv Ther. 2020 Jan;35(1):30-6.

18 Johansson JK, Niiranen TJ, Puukka PJ, Jula AM. Prognostic value of the variability in home-measured blood pressure and heart rate: the Finn-Home Study. Hypertension. 2012 Feb;59(2):212-8.

19 Horio M, Imai E, Yasuda Y, Watanabe T, Matsuo S. Modification of the CKD epidemiology collaboration (CKDEPI) equation for Japanese: accuracy and use for population estimates. Am J Kidney Dis. 2010 Jul;56(1):32-8.

20 Mancia G, Facchetti R, Parati G, Zanchetti A. Visit-to-visit blood pressure variability in the European Lacidipine Study on Atherosclerosis: methodological aspects and effects of antihypertensive treatment. J Hypertens. 2012 Jun;30(6):1241-51. 
21 Mancia G. Short- and long-term blood pressure variability: present and future. Hypertension. 2012 Aug;60(2): 512-7.

22 Asayama K, Kikuya M, Schutte R, Thijs L, Hosaka M, Satoh M, et al. Home blood pressure variability as cardiovascular risk factor in the population of Ohasama. Hypertension. 2013 Jan;61(1):61-9.

23 Chapter 2: definition, identification, and prediction of CKD progression. Kidney Int Suppl (2011). 2013 Jan; 3(1):63-72.

24 Parati G, Ochoa JE, Lombardi C, Bilo G. Assessment and management of blood-pressure variability. Nat Rev Cardiol. 2013 Mar;10(3):143-55.

25 Gansevoort RT, Correa-Rotter R, Hemmelgarn BR, Jafar TH, Heerspink HJ, Mann JF, et al. Chronic kidney disease and cardiovascular risk: epidemiology, mechanisms, and prevention. Lancet. 2013 Jul;382(9889): 339-52.

26 Seo SM, Chung WB, Choi IJ, Koh YS, Ihm SH, Kim PJ, et al. Visit-to-visit variability of systolic blood pressure predicts all-cause mortality in patients received percutaneous coronary intervention with drug-eluting stents. Heart Vessels. 2018 May;33(5):489-97.

27 Vidal-Petiot E, Stebbins A, Chiswell K, Ardissino D, Aylward PE, Cannon CP, et al. Visit-to-visit variability of blood pressure and cardiovascular outcomes in patients with stable coronary heart disease. Insights from the STABILITY trial. Eur Heart J. 2017 Oct; 38(37):2813-22.

28 Montalescot G, Sechtem U, Achenbach S, Andreotti F, Arden C, Budaj A, et al. 2013 ESC guidelines on the management of stable coronary artery disease: the Task Force on the management of stable coronary artery disease of the European Society of Cardiology. Eur Heart J. 2013 Oct;34(38):2949-3003.

29 Webb AJ, Fischer U, Mehta Z, Rothwell PM. Effects of antihypertensive-drug class on interindividual variation in blood pressure and risk of stroke: a systematic review and meta-analysis. Lancet. 2010 Mar;375(9718): 906-15. 